CCNY-HEP-00/5

hep-ph/0009225

\title{
Non-topological solitons as nucleation sites for cosmological phase transitions
}

\author{
D. METAXAS \\ Physics Department \\ City College of the City University of New York \\ New York, NY 10031 \\ metaxas@theory.sci.ccny.cuny.edu
}

\begin{abstract}
I consider quantum field theories that admit charged non-topological solitons of the Q-ball type, and use the fact that in a first-order cosmological phase transition, below the critical temperature, there is a value of the soliton charge above which the soliton becomes unstable and expands, converting space to the true vacuum, much like a critical bubble in the case of ordinary tunneling. Using a simple model for the production rate of Q-balls through charge accretion during a random walk out of equilibrium, I calculate the probability for the formation of critical charge solitons and estimate the amount of supercooling needed for the phase transition to be completed.
\end{abstract}




\section{Introduction}

A common feature of various cosmological models with a first-order phase transition is the generation of excessive entropy. This happens Because the probability of tunneling from the false to the true vacuum is negligible when the supercooling parameter $\eta=\left(T_{c}-T\right) / T_{c}$ is very small. The question arises then if there exists some other mechanism that can facilitate the completion of the transition more rapidly, without the problems of excessive entropy production.

In other cases of interest for cosmology a relatively large amount of supercooling is actually needed in order to have out-of-equilibrium processes such as the generation of baryon asymmetry. A detailed picture of the phase transition is then necessary.

Here I consider a different mechanism for a phase transition that is possible in theories that admit non-topological solitons of the Q-ball type, and show that the rate of their nucleation can be large enough for the transition to proceed with much less supercooling than in other cases.

There is a large amount of literature on non-topological solitons (see [1] for a review). Here I will be interested in the case of Q-balls that are formed in the context of a first-order cosmological phase transition.

The production of non-topological solitons in a cosmological phase transition has been studied before [2, 9], and I will now describe briefly some previous results, focusing in the case of a first-order transition.

Assume a potential $U(\phi, T)$ with a symmetry preserving minimum at $\phi=0$ and a symmetry breaking minimum at $\phi=\phi_{+}$, separated by a barrier with a local maximum $U_{M}$ at $\phi=\phi_{M}$, and consider the evolution of the vacuum during the phase transition. At high enough temperature $T$ the universe is in the symmetric vacuum. At temperatures close to the critical temperature $T_{c}$, thermal fluctuations of the field $\phi$ become important. The fluctuating regions typically have volume $V_{\xi}=\frac{4 \pi}{3} \xi^{3}$, where $\xi \sim \frac{1}{m_{\phi}(T)}$ is the correlation length. The relative probability of finding a certain configuration of the field $\phi$ in a correlation volume is $P \sim \exp (-F / T)$, where $F$ is the free energy of the given configuration.

For high enough temperatures thermal fluctuations are strong enough to flip regions of the correlation volume from the symmetric to the asymmetric minimum and vice-versa. When the temperature falls below a certain temperature called the Ginsburg temperature $T_{G}$, thermal fluctuations are not 
strong enough to mediate such transitions. Since the number density of the $\phi$ particles is $n_{\phi} \sim T^{3}$, we will have $N \sim T_{G}^{3} V_{\xi}$ charged particles in a correlation volume, and we expect a net charge fluctuation $Q_{b} \sim \sqrt{N}$ in each bubble of the asymmetric phase (I assume a case with zero initial charge asymmetry).

Usually, in theories that admit Q-balls, there is a constraint of a minimum charge $Q_{\min }$ that they have to exceed for reasons of stability. If $Q_{b}>Q_{\min }$ then we expect a Q-ball to be formed in the bubble of the asymmetric phase.

In the case of a first-order phase transition, when the symmetric vacuum becomes metastable, the potential energy density in the interior of the Qballs becomes negative with respect to the potential energy density of the symmetric vacuum. Then if a Q-ball has large enough charge it becomes unstable and expands, converting space to the true vacuum, much like a critical bubble in the case of ordinary tunneling [6, 5].

In previous discussions of Q-ball production in the early universe, the constraint of the minimum charge, was quite restricting for the occurence of the phase transition, especially since most of the estimates were made using the thin-wall approximation for the solitons, which leads to a relatively large minimum charge [4].

It was recently shown that the thin-wall assumption is not correct, and that some theories may admit Q-balls without the restriction of a minimum charge, or with a minimum charge of order one. Then, charge accretion may lead to a growth in the number density of the critical charge solitons below some temperature, and thus mediate the phase transition [5]. An estimation of the nucleation rate of these solitons is then important for the description of the phase transition.

In the case where the solitons that are initially produced survive long enough to reach thermal equilibrium, and if a set of reactions that produce critical solitons remain in thermal equilibrium, then critical solitons are copiously produced. The conditions for this to happen are quite model-dependent [5, 9]. Here I will deal with the case of critical charge Q-balls appearing before thermal equilibrium, and show that the rate of their production by charge accretion can be large enough for the phase transition to proceed. This case may be relevant also when we have soliton destruction or evaporation before they reach thermal equilibrium.

I will begin by reviewing various approximations that already exist for the description of Q-balls in various ranges of the charge $Q$. Then I will focus on the case of a first-order phase transition in theories that admit 
non-topological solitons and calculate the rate of Q-ball production using results from subcritical bubble fluctuations and charge fluctuations. At temperatures near the critical temperature thermal fluctuations may flip regions from the symmetric to the asymmetric vacuum. If these regions also contain a charge fluctuation $Q$, and have energy less than $Q$ free particles, then, instead of decaying into free particles, they will evolve into the soliton configurations. The evolution of these bubbles into solitons is affected by their interaction with the thermal environment. An absorption of a positive or negative charge can be simulated by a random walk with a + or - step. The difference from an unrestricted random walk is the existence of a minimum charge below which they disappear. This is the case of a random walk with an absorbing boundary. Using this model I will calculate the final soliton production rate. Once they are produced these solitons keep interacting with the medium, and their evolution can still be simulated by a random walk. Below the critical temperature there is also a critical soliton charge, above which the solitons become unstable. This is the case of a random walk with two absorbing boundaries. Using this model I will calculate the production rate of critical solitons. The critical solitons expand converting space to the true vacuum and mediating the phase transition. I will then estimate the amount of supercooling needed for the nucleation of a cosmological phase transition. The main result of this work is that, for generic values of the model's parameters, the phase transition can proceed much faster, with a much smaller amount of supercooling than other models (that do not admit nontopological solitons).

This result may be important for cosmological phase transitions in theories that admit non-topological solitons, such as the Minimal Supersymmetric Standard Model (MSSM) [5], [0], or phenomenological theories of hadronic matter [1].

In Sec. 2 I describe some approximations that already exist for the description of Q-balls in various regimes [3, 5, 6]. In Sec. 3 I will model the production of Q-balls during the phase transition, using results from subcritical bubble fluctuations [8] and charge fluctuations [9]. I will then calculate the probability for the formation of critical charge Q-balls through charge accretion and in Sec. 4 I will estimate the amount of supercooling needed for the completion of the phase transition. 


\section{Non-topological solitons in various approx- imations}

Consider a quantum field theory of a complex scalar field $\phi$ with Lagrangian

$$
L=\frac{1}{2}\left(\partial_{\mu} \phi\right)^{2}-U(\phi)
$$

that is admits a global $U(1)$ symmetry and a conserved charge

$$
\phi \rightarrow e^{i \theta} \phi, \quad Q=\frac{1}{2 i} \int d^{3} x \phi^{*} \stackrel{\leftrightarrow}{\partial_{t}} \phi
$$

Minimizing the energy in the sector of field configurations that have a given charge $Q$ yields the three-dimensional bounce equation

$$
\nabla^{2} \phi=U_{\omega}^{\prime}(\phi)
$$

where

$$
U_{\omega}(\phi)=U(\phi)-\frac{1}{2} \omega^{2} \phi^{2}, \quad \phi(x, t)=e^{i \omega t} \phi(x)
$$

This bounce equation has a solution when $U_{\omega}(\phi)$ has a nonzero global minimum in addition to the local minimum at the origin. In order for this to happen, $\omega$ must be less than $M$, where $M^{2}=U^{\prime \prime}(0)$, and greater than some value $\omega_{0}$, for which $U_{\omega}(\phi)$ has two degenerate minima. Minimizing $E_{\omega}$ with respect to $\omega$ we get the soliton energy $E_{Q}$.

I will be interested in the case of nontopological solitons that appear near a first-order phase transition for which I will consider a generic effective potential depending on the temperature $T$ :

$$
U(\phi, T)=\frac{1}{2} M^{2}(T) \phi^{2}-A(T) \phi^{3}+\lambda \phi^{4}
$$

where

$$
M^{2}(T)=\left(\alpha T^{2}-m^{2}\right)
$$

for which

$$
U_{\omega}(\phi)=\frac{1}{2}\left(M^{2}-\omega^{2}\right) \phi^{2}-A \phi^{3}+\lambda \phi^{4} .
$$

The cubic term is understood as an effective term of the form $\left(\phi^{*} \phi\right)^{3 / 2}$ that still has a global $U(1)$ symmetry, and $\alpha$ is the square of the gauge coupling 
constant, assumed to be greater than $\lambda$. The coefficient $A$ will also depend on the temperature, but I will neglect this dependence near the critical temperature.

In the limit of large $Q$, which is equivalent to $\omega \rightarrow \omega_{0}+0$, the form of the Q-ball solution simplifies considerably [3] since $U_{\omega}(\phi)$ is such that the thin wall approximation is valid for the bounce solution.

In the opposite limit of $\omega \rightarrow M$ we see that the barrier in the function $U_{\omega}(\phi)$, which provides the bounce solution, becomes very shallow until it disappears. In this approximation, neglecting the $\lambda \phi^{4}$ term, and after rescaling [5]

$$
\phi=\psi \frac{M^{2}-\omega^{2}}{A} \quad, \quad x=\xi \frac{1}{\sqrt{M^{2}-\omega^{2}}}
$$

one gets a minimum

$$
E_{Q}=Q M\left(1-\frac{\epsilon^{2}}{6}\right)
$$

if

$$
\epsilon \equiv \frac{Q A^{2}}{3 S_{\psi} M^{2}}<\frac{1}{2}
$$

where $S_{\psi}=4.85$ is the bounce action in the dimensionless potential $\frac{1}{2} \psi^{2}-\psi^{3}$. The bounce radius in $\xi$ is of order one so the soliton radius is

$$
R_{s} \sim \frac{1}{\sqrt{M^{2}-\omega^{2}}} \sim \frac{1}{\epsilon M}
$$

Now we consider the case where the previous potential is at, or slightly below, the critical temperature where it has two degenerate minima at 0 and $\phi_{+}$:

$$
U(\phi)=\lambda \phi^{2}\left(\phi-\phi_{+}\right)^{2}
$$

We consider large charge Q-balls in the thin wall approximation, but now the surface terms are important since the two minima are degenerate, and $\omega_{0}=0$ since the original potential has already a bounce solution.

The Q-ball energy in the thin wall limit is

$$
E(Q)=\frac{Q^{2}}{2 \phi^{2} V}+4 \pi R^{2} S_{1}-\varepsilon V
$$

where

$$
\varepsilon=\left|U\left(\phi_{+}\right)\right| \quad S_{1}=\int_{0}^{\phi_{+}} d \phi \sqrt{2 U(\phi)}
$$


An approximate expression for the radius if we neglect the potential term is

$$
R^{5}=\frac{9 Q^{2}}{(8 \pi)^{2} \phi^{2} S_{1}}
$$

As the temperature drops further from the critical temperature, the negative potential volume term becomes important, and there exists a value of $\varepsilon$ such that the Q-ball energy does not have a minimum, and is unstable against expansion. Equivalently, for a value of $\varepsilon$, there exists a critical value of the charge $Q$, such that for $Q>Q_{c}$ the Q-ball is unstable and expands converting space to the true vacuum. These critical solitons have a charge $Q_{c}$ and a radius $R_{c}$ which are obtained from the simultaneous solution of the equations $\frac{d E}{d R}=\frac{d^{2} E}{d R^{2}}=0$ to be [5]

$$
Q_{c}=\frac{400 \pi \sqrt{70}}{81} \frac{\phi_{+} S_{1}^{3}}{\varepsilon^{5 / 2}}
$$

and

$$
R_{c}=\frac{10}{3}\left(\frac{7}{8}\right)^{1 / 5} \frac{S_{1}}{\varepsilon}
$$

\section{$3 \quad$ Nucleation of critical Q-balls}

In this Section I will show that critical charge Q-balls can be produced through charge accretion from smaller charge Q-balls during a random walk out of equilibrium, and estimate the rate for this process.

I will use the generic finite temperature potential of the form (5). At the critical temperature $T_{c}$ we have

$$
V=\frac{1}{2} M^{2} \phi^{2}-A \phi^{3}+\lambda \phi^{4}=\lambda \phi^{2}\left(\phi-\phi_{+}\right)^{2}
$$

where $\phi_{+}$is the second degenerate minimum, and accordingly the parameters of the potential satisfy the relations

$$
M^{2}=2 \lambda \phi_{+}^{2}, A=2 \lambda \phi_{+}, M^{2}=A^{2} / 2 \lambda
$$

The surface term is

$$
S_{1}=\int_{0}^{\phi_{+}} d \phi \sqrt{2 V(\phi)}=\frac{\sqrt{2 \lambda}}{6} \phi_{+}^{3}
$$


and at a temperature $T=T_{c}-\delta T$ slightly below the critical temperature the potential energy difference of the two minima is

$$
\varepsilon=\left|V\left(\phi_{+}(T)\right)\right|=\alpha T \delta T \phi_{+}^{2}=\alpha T^{2} \phi_{+}^{2} \eta
$$

where $\eta=\delta T / T$ is the supercooling parameter.

I will estimate the production rate of Q-balls at the critical temperature using results of subcritical bubble calculations [8] and charge fluctuations [9]. Subcritical bubbles are bubbles of the symmetry breaking minimum that have smaller than the critical radius. In order to get an approximation for the production rate of subcritical bubbles we will use the ansatz for Gaussianshaped bubbles of radius $R$

$$
\phi_{b}(r)=\phi_{+} e^{-r^{2} / R^{2}}
$$

and write an approximate expression for their thermal production rate

$$
\Gamma^{\prime}(R)=a T^{4} e^{-F\left(\phi_{b}\right) / T}
$$

where $a$ is an unknown numerical factor of order one and $F\left(\phi_{b}\right)$ is the bubble free energy

$$
F\left(\phi_{b}\right)=\int 4 \pi r^{2} d r\left(\frac{1}{2}\left(\frac{d \phi}{d r}\right)^{2}+V(\phi, T)\right)=\gamma R+\delta R^{3}
$$

For a bubble of the form (21) and the potential (5) at the critical temperature, it turns out that the potential term will give the largest contribution to the production rate below, with a coefficient [8]

$$
\delta=\pi^{3 / 2} \phi_{+}^{2}\left(\frac{\sqrt{2}}{8} M^{2}-\frac{\sqrt{3}}{9} A \phi_{+}+\frac{\lambda}{8} \phi_{+}^{2}\right)=c_{1} \phi_{+}^{2} M^{2}
$$

where $c_{1}=0.26$.

Now, in a typical bubble of radius $R$ there are

$$
N \approx c_{2} T^{3} R^{3}
$$

$\phi$ and $\bar{\phi}$ particles, where $c_{2}=\frac{4 \pi \zeta(3)}{3 \pi^{2}}=0.51$. The probability of a fluctuation of total charge $Q$ in this number is [9]

$$
P(Q ; N)=\frac{e^{-Q^{2} / N}}{\sqrt{N}}
$$


Then, changing variables from $R$ to $N$, the total production rate of bubbles of charge $Q$ is

$$
\begin{aligned}
\Gamma^{\prime}(Q) & =\int \Gamma(N) P(Q ; N) d N= \\
& =3 \sqrt{c_{2}} a T^{11 / 2} \int e^{-\frac{c_{1} \phi^{2} M^{2} R^{3}}{T}-\frac{Q^{2}}{c_{2} T^{3} R^{3}}} \sqrt{R} d R
\end{aligned}
$$

where from now on we write $\phi$ for the symmetry breaking minimum $\phi_{+}$. We can evaluate this integral using the saddle point method. The exponent has a minimum at a radius

$$
R_{b}^{3}=\frac{Q}{\sqrt{c_{1} c_{2}} \phi M T} .
$$

Absorbing some numerical constants in the prefactor $a$ of order one, and denoting a new numerical constant $c=2 \sqrt{c_{1}} / \sqrt{c_{2}}=1.42$ we get the final result

$$
\Gamma^{\prime}(Q)=a \frac{T^{13 / 2}}{(\phi M)^{5 / 4} Q^{1 / 4}} e^{-c \frac{Q \phi M}{T^{2}}}
$$

The above expression for the nucleation rate $\Gamma^{\prime}(Q)$ of bubbles of the true vacuum with net charge $Q$ is still not quite the same as the nucleation rate of Q-balls. First, the bubble energy should be smaller than the corresponding energy of $Q$ free particles, otherwise the bubble will decay into free particles instead of evolving into a soliton. From our previous expressions we see that, for generic values of our parameters, this is indeed the case, and accordingly the charged bubbles, during their subsequent interaction with the particles of the heat bath, will preferably evolve to the soliton configuration instead of decaying into free particles. Although the initial bubble energy is not exactly the same as the corresponding soliton energy, the thermal fluctuations from the surrounding heat bath would rapidly compensate for that during the subsequent random walk. It should be noted, however, that the fact that the bubble energy is less than the corresponding energy of $Q$ free particles is also a model-dependent condition and has to be checked in different models.

Second, the Q-balls are bounces in a modified potential, and may be very different configurations from the true vacuum bubbles. If, for example, the bubble radius is much smaller than the corresponding soliton radius then it will expand until it becomes the lowest energy configuration of a Q-ball, but in the process it will meet a number of particles contained in this volume, 
and if there is a net charge fluctuation of negative charge $Q$, then the bubble charge will disappear. From the expressions for the corresponding radii of the subcritical bubbles and the solitons, (25) and (11), and using (10) we get for the ratio of the volumes of the bubble and the soliton of charge Q:

$$
\left(\frac{R_{b}}{R_{s}}\right)^{3} \sim \frac{Q}{\sqrt{c_{1} c_{2}} \phi M T} \epsilon^{3} M^{3}=40 \epsilon^{4} \frac{M^{4}}{A^{2} \phi T}
$$

From (18) we have:

$$
M^{2} \sim \alpha T^{2} \sim \lambda \phi^{2}, A \sim \alpha \phi
$$

thus

$$
\left(\frac{R_{b}}{R_{s}}\right)^{3} \sim 40 \epsilon^{4} \sqrt{\frac{\alpha}{\lambda}}
$$

with

$$
\epsilon \sim Q A^{2} / M^{2} \sim Q \lambda
$$

and for charges of order one we see that $\epsilon$ is much less than one, in which case we indeed have the situation mentioned above.

During the expansion of the bubble to a soliton, the bubble will encounter a number of positive and negative charges $N$ which are contained in the volume of the soliton. This process can be simulated by a random walk, with $\mathrm{a}+(-)$ step if a Q-ball absorbs a positive (negative) charge. The difference from an unrestricted random walk is the existence of a minimum charge below which the Q-balls are unstable and disappear, This is the situation in a random walk with an absorbing boundary, and the probability that an initial charge $Q^{\prime}$ will evolve to a charge $Q$ is given by [10]:

$$
P\left(Q^{\prime}, Q\right)=\frac{Q Q^{\prime}}{N^{3 / 2}} e^{-\frac{Q^{2}+Q^{\prime 2}}{2 N}}
$$

where $N$ is the number of particles contained in the soliton volume (from now on we will use $Q$ and $Q^{\prime}$ to denote the units of charge above the minimum charge $Q_{\text {min }}$ ).

Hence, the final expression for the nucleation rate of solitons of charge $Q$ is

$$
\Gamma(Q)=\int_{0}^{\infty} \Gamma^{\prime}\left(Q^{\prime}\right) P\left(Q^{\prime}, Q\right) d Q^{\prime}
$$


Using the previous equations (26), (31), (11), we get the final result for the nucleation rate of solitons of charge $Q$

$$
\Gamma(Q)=\Gamma_{m} \frac{\lambda^{9 / 2} M^{5 / 2}}{T^{1 / 2} \phi^{2}} Q e^{-\frac{2 \lambda^{3} M^{3}}{T^{3}} Q^{2}}
$$

where

$$
\Gamma_{m}=a \frac{T^{13 / 2}}{(\phi M)^{5 / 4}} e^{-c \frac{Q_{\min } \phi M}{T^{2}}}
$$

and again we absorbed numerical factors of order one in the prefactor $a$.

Once the Q-balls are formed and start interacting with the positive and negative charges in the heat bath, their subsequent evolution, before they reach thermal equilibrium or disappear, can be simulated again by a random walk, with $\mathrm{a}+(-)$ step if a Q-ball absorbs a positive (negative) charge. The difference from an unrestricted random walk besides the existence of a minimum charge below which the Q-balls are unstable and disappear, is the existence of a critical charge above which the Q-balls are unstable and expand converting space into the true vacuum. This is the situation in a random walk with two absorbing boundaries.

We are interested in the probability for a Q-ball, starting from an initial charge $Q_{0}$ which is arbitrary but small enough so that the rate for its nucleation is not negligible, to reach the critical charge $Q_{c}$ before it reaches the minimum charge, and we also need an estimate of the total time that is needed for this process.

Let $X(Q, N)$ be the probability that a point at initial position $Q$ will have crossed $Q_{c}$ by $N$ steps before crossing 0 . Let $p$ and $q$ be the relative probabilities for up and down motion in a single step, with $p+q=1$. For sufficiently large number of steps $X(Q, N)$ becomes independent of $N$. In this case it is easy to calculate this probability $X(Q)$. Assuming there is no asymmetry between positive and negative charges, and $p=q=1 / 2$, we get円 $[10]$

$$
X(Q)=\frac{Q}{Q_{c}}
$$

and the nucleation rate for critical charge solitons is

$$
\Gamma\left(Q_{c}\right)=\int d Q X(Q) \Gamma(Q)
$$

\footnotetext{
${ }^{1}$ Note that we have shifted the charges by $Q_{\min }$ so the exact expression for (35) would be $X(Q)=\frac{Q}{Q_{c}-Q_{\min }}$ but since $Q_{c} \gg Q_{\min }$ the above expression (35) would suffice.
} 
For applications to a realistic situation we are interested in a different kind of random walk, one where the time step is variable, depending on the value of the Q-ball charge at any step, since different charges have different cross sections. If $n(\phi)$ and $v$ are the density and velocity of the particles in the thermal bath and $\sigma(Q)$ is the cross section for their absorption by a Q-ball of charge $Q$, then the time step varies as

$$
\Delta t \sim \frac{1}{n(\phi) \sigma(Q) v}
$$

As a first approximation for $\sigma(Q)$ we can take

$$
\sigma(Q) \sim 4 \pi R_{Q}^{2}
$$

where $R_{Q}$ is the radius of the Q-ball. During a random walk from a small to a critical Q-ball this radius varies by several orders of magnitude, and also with different dependence on the charge, according to relations such as (11), (15).

However, the simple result for the probability (35) is derived in the limit of a large number of steps $N$ and turns out to be finite and independent of $N$. It is the same whether we use the smallest or the largest time step, as long as the number of steps is large enough, and so it must be the same even if the time step is varying.

I have performed several numerical trials of random walks with initial charge $Q_{0}$ and critical charge $Q_{c}$, and time steps with various dependences on the charge and indeed it turns out that for any sample of random walks there is always a fraction of $Q_{0} / Q_{c}$ of them that cross the critical charge, the dependence on time is very similar to the dependence on the number of steps, and they are all completed within $Q_{c}^{2}$ steps. Some examples of these random walks are shown in Fig. 1 and Fig. 2.

In these examples I used the numerical values of $Q_{0}=10, Q_{c}=300$, and for a sample of 3000 trials of random walks, a fraction of $Q_{0} / Q_{c}$ where successful (crossed the critical charge). They where all completed within $Q_{c}^{2}$ steps and I show the results in Fig. 1. I used a radius-charge dependence $R_{Q} \propto Q^{2 / 5}$, for which the time step varies as $\Delta t \propto Q^{-4 / 5}$, and I show the results for the duration of these random walks in Fig. 2.

The assumption of a geometric cross section as an estimate for $\sigma(Q)$ is justified by recent results [11], at least for large values of the charge. From our 
numerical trials of random walks we see that indeed the successful random walks spend most of the time in large values of the charge, so we expect it to be a good approximation.

Another approximation that was implicit in the random walk model was that the absorption of a positive (negative) charge leads instantaneously to a Q-ball of charge $Q \pm 1$. Actually, it leads to an excited Q-ball of charge $Q \pm 1$ according to reactions like

$$
(Q)+\phi \rightarrow(Q+1)+\Delta E(Q) .
$$

However, one can easily see that generally $\Delta E(Q)$ is less than $M$, the elementary mass of the particles, and using (37), (38) one can see that the thermal fluctuations coming from collisions with the surrounding particles

of the heat bath during an elementary time step of the random walk are of order $T$ which is generally much greater than $M$, hence these collisions bring rapidly an excited Q-ball to its ground state.

\section{Nucleation of cosmological phase transitions}

From the previous expressions for the critical charge and the critical radius, Eqs. (16), (17), and using the relations for our general finite temperature potential slightly below the critical temperature, Eqs. (18), (19), (20), we get

$$
Q_{c}=\frac{c_{3}}{\lambda}\left(\frac{M}{\sqrt{\alpha} T}\right)^{5} \frac{1}{\eta^{5 / 2}}, \quad R_{c}=c_{4} \frac{\lambda^{1 / 2}}{\alpha} \frac{\phi}{T^{2} \eta}
$$

with numerical constants $c_{3}=0.3, c_{4}=0.7$. For the parameters of the potential $M, A, \phi_{+}$, I will use their values at the critical temperature, assuming as will be shown later that the transition happens at a small value of the supercooling parameter. For numerical results I will take the coupling constants $\lambda=0.1, \alpha=0.2$, and the scale of the phase transition at the temperature $T_{c}=1 T e V$.

I will assume a Friedman-Robertson-Walker (FRW), radiation-dominated universe, with the time-temperature relation

$$
t=\frac{M_{P}}{T^{2}}
$$


where

$$
M_{P}=\sqrt{\frac{45}{16 \pi^{3} N}} M_{\text {Planck }}
$$

and $N=N_{b}+\frac{7}{8} N_{f}$ are the essentially massless degrees of freedom. Thus, for temperature $T=T_{c}-\delta T$ slightly below the critical temperature the supercooling parameter is

$$
\eta=\frac{\delta T}{T}=\frac{\delta t}{2 t}
$$

I will estimate the value of the supercooling parameter at which there are enough critical Q-balls produced, at least one per Hubble volume, in order to induce the phase transition [13.

The critical charge is infinite at and above the critical temperature. There are subcritical bubbles and solitons produced at these temperatures but the probability for their survival below the critical temperature is exponentially small, since this evolution is equivalent to a random walk with one boundary and for this process we expect an initial charge $Q$ to disappear after $N \sim Q^{2}$ collisions.

Let $\tau\left(Q_{c}\right)$ be the time needed for the completion of the random walk for a critical charge $Q_{c}$. Then if

$$
\tau\left(Q_{c}\right) \frac{d Q_{c}}{d t} \ll Q_{c}
$$

the variation of the critical charge during the random walk is irrelevant, and the number density of the critical Q-balls produced at some time is

$$
n\left(Q_{c}, t\right)=\int d Q d t^{\prime} \frac{Q}{Q_{c}\left(t^{\prime}\right)} \Gamma\left(Q, t^{\prime}\right)
$$

where $Q / Q_{c}$ is the probability that a critical Q-ball with charge $Q_{c}$ will be nucleated starting from a Q-ball with charge $Q . \Gamma\left(Q, t^{\prime}\right)$ is the nucleation rate of Q-balls at time $t^{\prime}$, and for a fast transition I will approximate it with the nucleation rate $\Gamma(Q)$ at the critical temperature (26).

From the previous results for the random walk we can also get an estimate for $\tau\left(Q_{c}\right)$

$$
\tau\left(Q_{c}\right) \simeq Q_{c}^{2} \Delta t
$$


where, for relativistic particles with density $n(\phi)$ in the heat bath, and cross section for absorption $\sigma(Q) \simeq 4 \pi R_{Q}^{2}$, the time step is

$$
\Delta t \simeq \frac{1}{n(\phi) \sigma(Q)} \simeq \frac{1}{T^{3} R_{Q}^{2}}
$$

For an order of magnitude estimate of $\tau\left(Q_{c}\right)$ I consider an average charge $Q=Q_{c} / 2$, with the radius-charge dependence given by (15), since it is near the phase transition but far from the critical charge:

$$
R_{Q}^{2} \simeq \frac{1}{\lambda^{1 / 5}} \frac{Q^{4 / 5}}{\phi^{2}}
$$

Also we have

$$
\frac{d Q_{c}}{d t}=\frac{d Q_{c}}{d \eta} \frac{d \eta}{d t}
$$

where, from (41)

$$
\frac{d \eta}{d t}=\frac{T^{2}}{2 M_{P}}
$$

Using (40), (45), (46), (47), (48), (49), the condition for the validity of our approximation (43) becomes:

$$
\begin{gathered}
\tau\left(Q_{c}\right) \frac{d Q_{c}}{d t} \ll Q_{c} \Leftrightarrow \\
\Leftrightarrow Q_{c}^{2} \frac{1}{T^{3}} \frac{\lambda^{1 / 5} \phi^{2}}{\left(Q_{c} / 2\right)^{4 / 5}} \frac{T^{2}}{2 M_{P}} \frac{5 Q_{c}}{2 \eta} \ll Q_{c} \Leftrightarrow \\
\Leftrightarrow \eta^{4} \gg \frac{1}{\lambda}\left(\frac{M}{\sqrt{\alpha} T}\right)^{6} \frac{\phi^{2}}{T M_{P}}
\end{gathered}
$$

With $M_{P} \sim 10^{19} \mathrm{GeV}$ and for a phase transition at a scale $T \sim \phi \sim 1 \mathrm{TeV}$, this condition can be satisfied for a small value of the supercooling parameter $\eta$. If this condition holds, then

$$
\begin{aligned}
n\left(Q_{c}, t\right) & =\int d Q d t^{\prime} \frac{Q}{Q_{c}\left(t^{\prime}\right)} \Gamma\left(Q, t^{\prime}\right)= \\
& =\int_{0}^{\infty} Q \Gamma(Q) d Q \int_{0}^{\eta} \frac{\lambda\left(\eta^{\prime}\right)^{5 / 2}}{c_{3}} \frac{2 M_{P}}{T^{2}}\left(\frac{\sqrt{\alpha} T}{M}\right)^{5} d \eta^{\prime}
\end{aligned}
$$


Here I took the lower limit of the time integral at the critical temperature, assuming that the condition (50) is quickly satisfied.

From the previous expression (33) for $\Gamma(Q)$ we have

$$
\int_{0}^{\infty} Q \Gamma(Q) d Q=a \frac{T^{21 / 2}}{(\phi M)^{13 / 4}} e^{-c \frac{\phi M}{T^{2}} Q_{\min }}
$$

so we get

$$
n\left(Q_{c}, t\right)=a \frac{T^{17 / 2}}{(\phi M)^{13 / 4}} e^{-c \frac{\phi M}{T^{2}} Q_{\min }}\left(\frac{\sqrt{\alpha} T}{M}\right)^{5} \lambda M_{P} \eta^{7 / 2}
$$

where, $\phi=\phi_{+}$is the asymmetric minimum at the critical temperature, and $M=M\left(T_{c}\right)$. Thus, the condition for a large enough density of Q-balls to be produced inside a Hubble volume in order to mediate the phase transition:

$$
n\left(Q_{c}, t\right) \gtrsim\left(\frac{T^{2}}{M_{P}}\right)^{3}
$$

gives

$$
a e^{-c \frac{\phi M}{T^{2}} Q_{\min }} \eta^{7 / 2} \gtrsim\left(\frac{T}{M_{P}}\right)^{4} \frac{1}{\lambda}\left(\frac{\phi M}{T^{2}}\right)^{13 / 4}\left(\frac{M}{\sqrt{\alpha} T}\right)^{13 / 4}
$$

with the numerical constant $c=1.42$ and $a$ a numerical factor of order one.

If $Q_{\min }$ is small enough for this condition to be satisfied, then the transition proceeds at the corresponding value of $\eta$. Again, because of the factors of $M_{P}$, for a late time phase transition at the scale of $1 \mathrm{TeV}$, this condition is satisfied for a very small value of the supercooling parameter, if the minimum charge is small enough.

The nucleation of a sufficient number of critical Q-balls depends sensitively on the minimum charge.We see that for large enough $Q_{\min }$ (greater than 22 in our example) the phase transition cannot proceed through Q-ball nucleation, at least not in the range of our approximations, something which is consistent with our expectations and with previous results 4 .

As $Q_{\min }$ becomes smaller, there is a sufficient number of critical Q-balls produced at small values of the supercooling parameter.

For example, for $Q_{\min }=20$, we see that there are enough critical Q-balls produced per Hubble volume to nucleate the phase transition at values of the supercooling parameter $\eta=\frac{\delta T}{T} \sim 10^{-2}$. 
At these values of $\eta$, if we consider the case of ordinary tunneling we find that the tunneling rate is much smaller than the expansion rate of the Universe, and the transition does not happen until much later times and larger values of the supercooling parameter. Indeed, in our range of values of the supercooling parameter $\eta \sim 10^{-2}$, the nucleation rate of ordinary critical bubbles at high temperature is calculated in the thin-wall approximation 12 $\Gamma \sim T^{4} e^{-B}$, where

$$
B=\frac{S_{1}^{3}}{3 \varepsilon^{2} T} \sim \frac{\sqrt{\alpha}}{\lambda} \frac{1}{\eta^{2}}
$$

and we see that, for $\eta \lesssim 10^{-1}$, and for our values of parameters $\alpha$ and $\lambda$, the result for the ordinary tunneling rate is indeed exponentially smaller than the Hubble expansion rate.

For a better description of the phase transition one needs to calculate quantities like $p(t)$, the fraction of the coordinate volume of the universe that remains in the symmetric phase $\phi=0$ at time $t$ after the beginning of the phase transition, which in our case may start right after the time $t_{c}$ when the temperature has dropped to the critical temperature $T_{c}$. The usual formula given in 14 can be easily generalized to take into account the initial critical soliton radius, and the velocity of the soliton expansion:

$$
\ln p(t)=-\int_{t_{c}}^{t} d t_{1} \Gamma\left(Q_{c}, t_{1}\right) R^{3}\left(t_{1}\right) \frac{4 \pi}{3}\left(\frac{R_{c}\left(t_{1}\right)}{R\left(t_{1}\right)}+\int_{t_{1}}^{t} d t_{2} \frac{v\left(t_{2}\right)}{R\left(t_{2}\right)}\right)^{3}
$$

Here, $R$ is the scale factor of the Robertson-Walker metric, $R_{c}$ is the critical soliton radius, and $v$ is the velocity of the critical soliton expansion. The nucleation rate of critical solitons $\Gamma\left(Q_{c}, t_{1}\right)$ and their initial radius $R_{c}\left(t_{1}\right)$ are calculated from (36), (40). Not much is known about the velocity of expansion of critical solitons. We do not expect it to be close to the speed of light, since we are very close to the critical temperature and the amount of supercooling is very small. However, if we assume that the expansion is similar to the expansion of a true vacuum bubble in the case of an ordinary first-order phase transition, then we can use a simple model to approximate it by balancing the pressure inside and outside the bubble [12]

$$
v=c_{5} \frac{\varepsilon}{T^{4}}=c_{5} \frac{\alpha \phi^{2}}{T^{2}} \eta
$$

where $c_{5}=30 / \pi^{2}$. 
Substituting from the previous results, and keeping the first-order correction coming from the initial soliton radius, we get

$$
\begin{aligned}
\ln p(\eta)=- & a e^{-c \frac{\phi M}{T^{2}} Q_{\min }}\left(\frac{T^{2}}{\phi M}\right)^{5 / 4}\left\{\lambda \alpha^{11 / 2}\left(\frac{M_{P}}{T}\right)^{4} \frac{T^{3} \phi^{4}}{M^{7}} \eta^{17 / 2}\right. \\
& \left.+\lambda^{3 / 2} \alpha^{7 / 2}\left(\frac{M_{P}}{T}\right)^{3} \frac{T^{4} \phi^{3}}{M^{7}} \eta^{11 / 2}\right\}
\end{aligned}
$$

Here, the first term in the brackets would be the final result if we had neglected the initial soliton radius. The second term is the first-order correction that comes from the critical soliton size.

This result confirms our previous estimates for the nucleation of the phase transition. The mechanism is similar to inhomogeneous nucleation or nucleation in the presence of impurities [15]. The corrections coming from the critical soliton size are negligible for generic values of the couplings in our model, but for exceptional values of the couplings, or maybe different models, they would have to be taken into account.

\section{Conclusion}

The final results (53), (55), (59) depend quite sensitively on the minimum soliton charge allowed for stability. Thus the relaxed minimum bound on the charge described in Section 2 is important. As explained before, the minimum charge that the solitons have to exceed for reasons of stability is a model dependent parameter, and our results for the occurence or not of the phase transition can also be viewed as conditions on this charge in order for the transition to proceed via Q-ball nucleation.

I used a simplified model for the soliton production based on charge fluctuations, simulating the absorption of positive or negative charges by a random walk and ignoring other processes like soliton evaporation that may be included in more complete models. I assumed that there is no asymmetry between the Q-ball interaction with positive and negative charges, and also that the absorption of a positive (negative) charge by the Q-ball turns it instantaneously into a soliton of charge $Q \pm 1$. I also assumed a geometrical cross-section for absorption of elementary particles by the Q-ball, which

should be a valid approximation for macroscopic Q-balls of large charge. For 
small charge this may break down but since the Q-balls spent most of the time of the random walk in large values of the charge this should be a valid approximation.

Although these approximations are made to simplify the analysis, I do not expect the final results for the minimum soliton charge and the contribution to the phase transition to change significantly if appropriate corrections are included, since these modifications may only affect the pre-exponential factors in (53).

The most important correction is probably concerned with the model of soliton production via fluctuations of true vacuum bubbles. This is not as well-tested as other mechanisms of soliton production like, for example, the Kibble mechanism [16], but there are indications that the subcritical bubble formalism is correct [8].

\section{Acknowledgments}

I would like to thank Profs. E. J. Weinberg and V. P. Nair for many useful comments. This work was supported in part by a PSC-CUNY award. 


\section{References}

[1] T.D. Lee and Yang Pang, Phys. Rept. 221, 251 (1992).

[2] J.A. Frieman et al., Phys. Rev. Lett. 60, 2101 (1988).

[3] S. Coleman, Nucl. Phys. B262, 263 (1985).

[4] J. Ellis, K. Enqvist, D.V. Nanopoulos, K.A. Olive, Phys. Lett. 225B, 313 (1989).

[5] A. Kusenko, Phys. Lett. 406B, 26 (1997).

[6] D. Spector, Phys. Lett. 194B, 103 (1987).

[7] K. Enqvist and J. McDonald, Phys. Lett. 425B, 309 (1998).

[8] M. Gleiser, E.W. Kolb and R. Watkins, Nucl. Phys. B364, 411 (1991).

[9] K. Griest, E.W. Kolb and A. Massarotti, Phys. Rev. D40, 3529 (1989).

[10] W. Feller, An introduction to probability theory and its application, Vol. 1, Wiley (1990).

[11] T. Multamaki and I. Vilja Phys. Lett. 484B, 283 (2000).

[12] A.D. Linde, Nucl. Phys. B216, 421 (1983).

[13] A. Guth and E.J. Weinberg, Phys. Rev. D23, 876 (1981).

[14] A. Guth and S.H.H. Tye Phys. Rev. Lett. 44, 631 (1980).

[15] M.B. Christiansen and J. Madsen Phys. Rev. 53, 5446 (1996).

P. Shukla, A.K. Mohanty, S.K. Gupta, M. Gleiser, hep-ph/0006071.

[16] T. W. B. Kibble, J. Phys. A 9, 1387 (1976). 


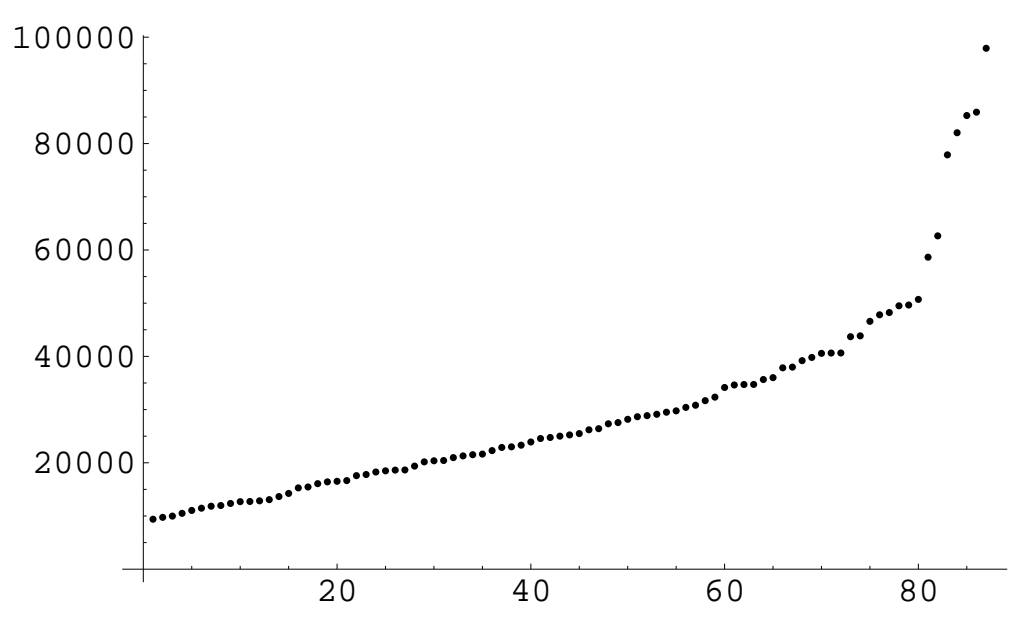

Figure 1: In the vertical axis we plot the number of steps of the random walk, and in the horizontal axis the successful trials that cross the critical charge.

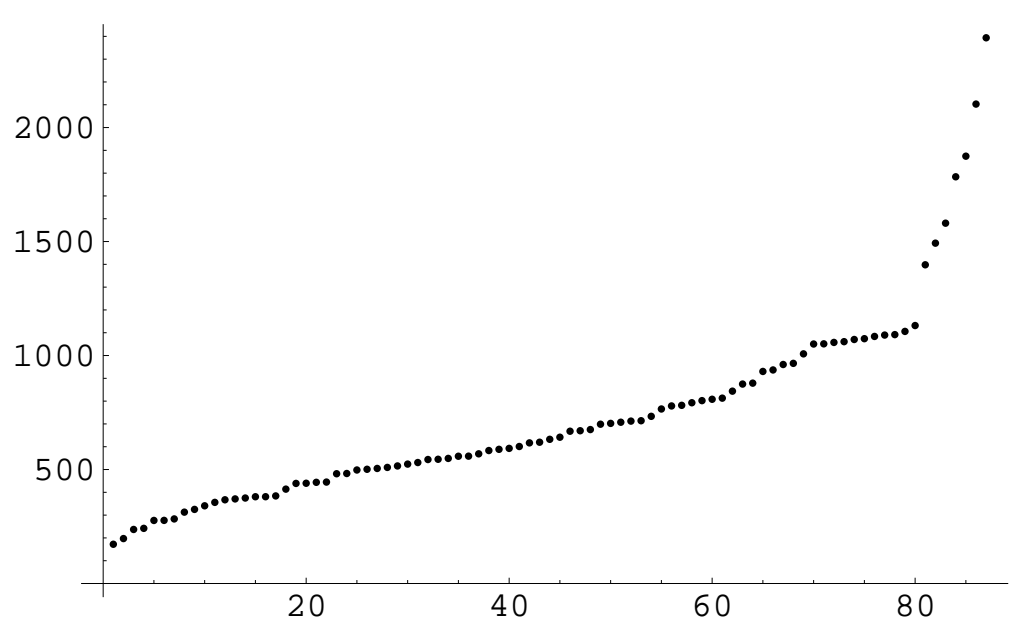

Figure 2: In the vertical axis we plot the duration time of the random walk in units of $\phi^{2} / T^{3}$ and in the horizontal axis the successful trials. 